\title{
Use of Aberration-Corrected STEM for Direct Structure and Chemistry Analysis of Catalytic Metal Particles
}

\author{
W. Sinkler*, S. A. Bradley*, L. F. Allard** and P. M. Voyles*** \\ * UOP LLC, 200 E. Algonquin Rd., Des Plaines IL 60017-5017. \\ ** Materials Science and Technology Div., Oak Ridge National Laboratory, Oak Ridge, TN 37831 \\ *** University of Wisconsin, Madison, Materials Science and Engineering, Madison, WI 53706.
}

The advent of probe-based aberration corrected STEM imaging presents intriguing new possibilities for characterization of catalytic materials. Imaging of single high-Z atoms in catalysts, such as Pt on a light element support, is now routinely achieved. This may provide the basis for directly determining the structures of catalytic fine metal particles [1]. Sensitivity to atomic number in HAADF-STEM images also suggests that fine metal particle chemistry may be accessible using atomic-resolution HAADF-STEM images. In order to determine the feasibility of such measurements a rigorous treatment of recorded intensities in atomic-resolution images is needed. This may also provide new insights into the experiment itself, possible artifacts, and how best to tune the experiment to obtain reliable information.

The current paper describes a methodology for analyzing aberration-corrected HAADF-STEM images which can aid in differentiating elements, and provide a direct estimate of the number of atoms in a fine metal cluster. Experimental dark-field STEM images of isolated atoms are crosscorrelated and averaged to obtain a motif representing the average intensity and shape of an isolated atom. By then cross-correlating this motif with image locations containing isolated atoms, the intensity of each atom is quantified in order to assess differentiation of elements. To analyze a cluster containing many atoms which do not necessarily line up well along the beam direction, the total dark field intensity after background subtraction is compared with the average intensity of isolated atoms in the same image or one taken under identical conditions. This provides in principle an estimate of the number of atoms in the cluster (for monometallic clusters).

Two types of industrially significant catalysts were considered: $\mathrm{Pt} / \mathrm{Al}_{2} \mathrm{O}_{3}$ and Pt-Element $\mathrm{A} / \mathrm{Al}_{2} \mathrm{O}_{3}$ (element $\mathrm{A}$ is a $4 \mathrm{~d} / 5 \mathrm{p}$ range element). Both types of samples were imaged using probe-corrected STEM instruments operated at $200 \mathrm{kV}$. Fig. 1 shows an example of a $\mathrm{Pt} / \mathrm{Al}_{2} \mathrm{O}_{3}$ catalyst with both $\mathrm{Pt}$ clusters and isolated Pt atoms. In Fig. 2 the histogram of the single-atom intensities analyzed as described above is shown and is compared to a histogram obtained from an image of Pt-A/ $\mathrm{Al}_{2} \mathrm{O}_{3}$. While the Pt-A image in this example does not show a bimodal histogram, the difference in the histograms' breadths may reflect the presence of the lighter A atoms in addition to Pt.

The total intensity above background for the cluster at center left of Fig. 1 corresponds to about 40 times the average intensity of isolated atoms in the same image, and the highest intensity in the cluster corresponds to about 3.6 times the average atom intensity. This suggests that there are approximately 40 atoms in the cluster, and that the cluster shape is slightly flattened against the support, since the cluster width corresponds to 6-8 atom diameters, while the thickness along the beam is between 3 and 4 atom diameters. 
The evidence shown here supports the feasibility of using Cs-corrected HAADF-STEM images for direct structure analysis of fine metal clusters in industrial catalysts. A reasonable estimate of the number of atoms in a Pt cluster was obtained, and preliminary evidence was shown for detection of two atom types using single-atom intensities. Further work is needed to optimize experimental conditions (voltage and camera length) and the analytical approach in order to obtain the maximal structural and chemical information from supported metal catalysts using Cs corrected STEM [2].

\section{References}

[1] V. Ortalan et al., Nature Nanotechnology. 5 (2010) 843.

[2] Microscopy research at the Oak Ridge National Laboratory's High Temperature Materials Laboratory was sponsored by the U. S. Department of Energy, Office of Energy Efficiency and Renewable Energy, Vehicle Technologies Program.

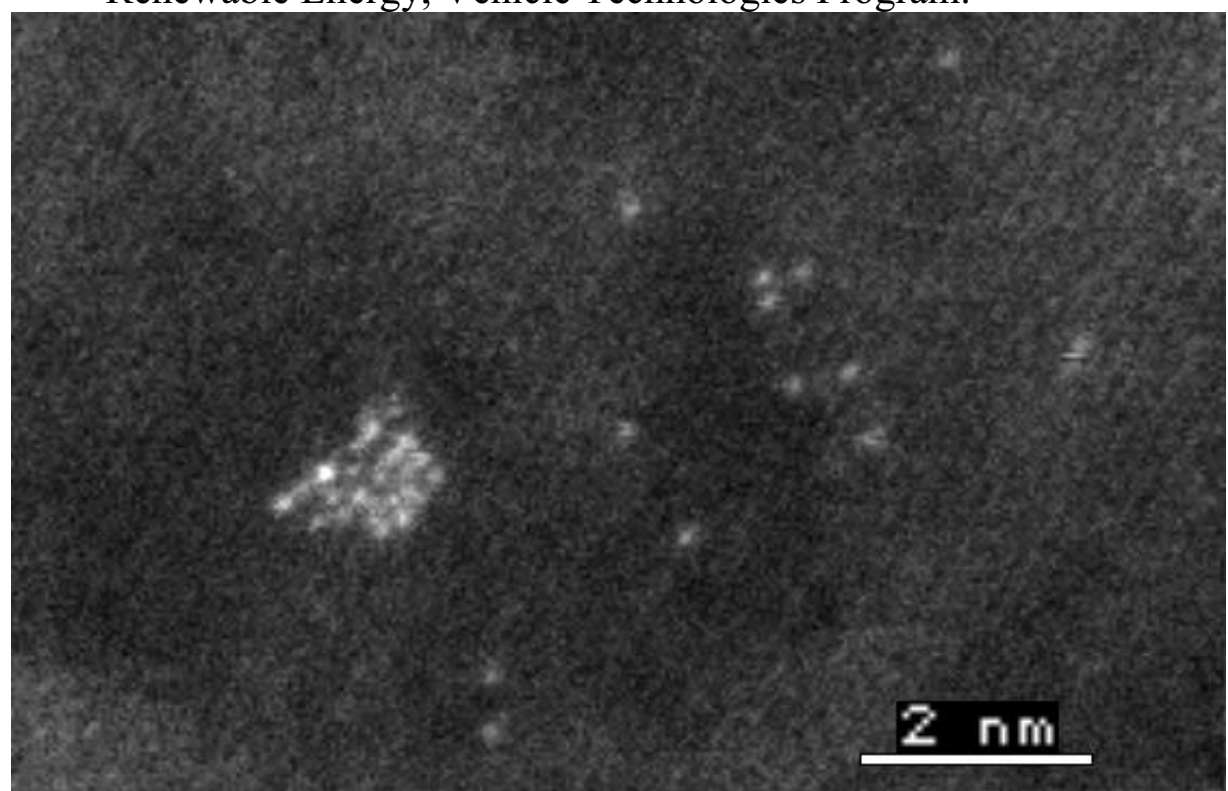

Fig. 1. Cs corrected HAADF-STEM image of Pt atoms and Pt cluster on alumina support
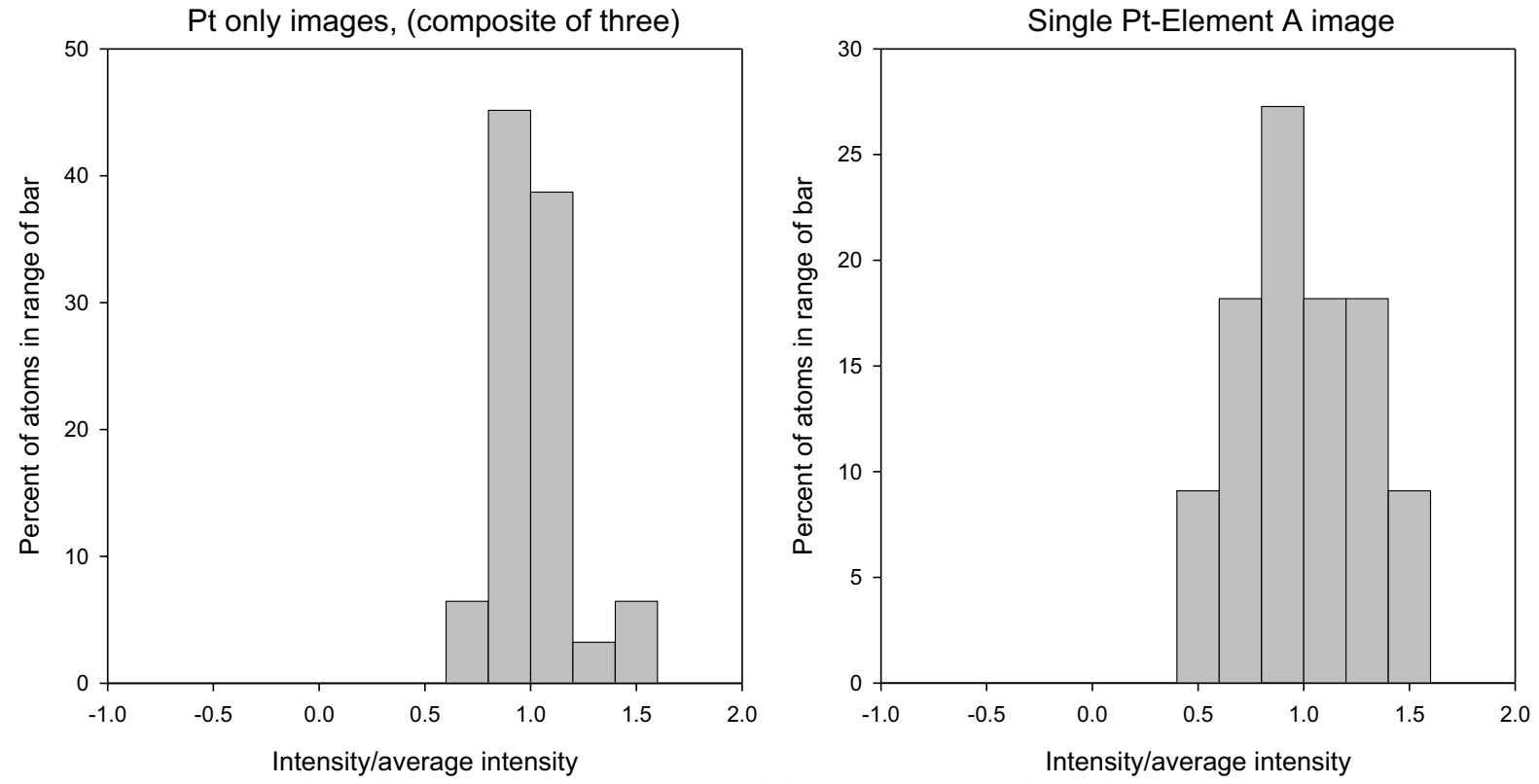

Fig. 2, Histograms of single-atom intensities from Pt/alumna images, and a Pt-A on alumina image. 